\title{
Submicrovillar Tubules in Distal Segments of Squid Photoreceptors Detected by Rapid Freezing
}

\author{
John P. Walrond' and Ete Z. Szuts ${ }^{2,3}$ \\ 'Department of Anatomy and Neurobiology, Colorado State University, Fort Collins, Colorado 80523, ${ }^{2}$ Marine Biological \\ Laboratory, Woods Hole, Massachusetts 02543, and ${ }^{3}$ Department of Physiology, Boston University School of Medicine, \\ Boston, Massachusetts 02118
}

Invertebrate phototransduction is believed to involve an inositol trisphosphate (Ins $\mathrm{P}_{3}$ )-mediated release of calcium from intracellular storage compartments. Although light-induced production of Ins $\mathrm{P}_{3}$ has been demonstrated for squid retinas, morphological evidence for the presence of internal calcium stores has been lacking. Because squid retinas are about 1 $\mathrm{mm}$ thick and composed of densely packed receptor cells, conventional aldehyde fixatives may not penetrate rapidly enough to preserve subcellular organelles. To reduce the time for fixative penetration, receptor cells were isolated from intact retinas before fixation, but these techniques provided little improvement in the preservation of membranebound compartments. Alternatively, the distal ends of the receptors were ultra-rapidly frozen by dropping $1 \mathrm{~mm}^{2}$ pieces of intact retina against a liquid helium-cooled copper block. Electron micrographs of thick sections from rapidly frozen and freeze-substituted retinas showed elongated saccules oriented parallel to the long axis of the receptor cell and located about $\mathbf{4 0} \mathbf{~ n m}$ from the microvillar openings. Freeze-fracture and etch views of rapidly frozen cells showed that the saccules are $130 \mathrm{~nm}$ diameter tubules and extend for at least several micrometers along the length of the receptor cell. We call these organelles submicrovillar tubules (SMT). The gap between the SMT and the plasma membrane contains a network of filaments that appear to be actin. Freeze-fracture and etch views of the rhabdomeres also indicate that adjacent microvilli are separated by a 6-8-nmwide extracellular space along most of their length. This space is spanned by extracellular connections linking adjacent microvilli. The position and orientation of the SMT suggest that these organelles may serve the same function as the more voluminous and highly convoluted submicrovillar cisternae found in other invertebrates. The SMT is likely to be the intracellular compartment that stores and releases calcium as part of the $\operatorname{Ins}_{3}$-mediated light response.

\footnotetext{
Received Aug. 16, 1991; revised Nov. 4, 1991; accepted Nov. 18, 1991

We thank Dr. Thomas S. Reese for the use of the rapid-freezing instrument, Dr. Susan Wood for assistance in the initial experiments, and Ms. Sara Huestis for technical assistance. We also thank the Integrated Microscopy Resource for Biomedical Research at the University of Wisconsin, Madison, for use of the highvoltage electron microscope and for the assistance of Dr. Hans Ris in the use of this instrument. Finally, we thank Drs. Steven Chamberlain, Maria Gomez, John Lisman, Enrico Nasi, Richard Payne, and Susan Wood for their instructive comments on an early version of this report. This research was initiated when J.P.W. was an NIH Staff Fellow. This research was supportcd in part by a BRSG grant and a National Institutes of Health grant (NS25572) to J.W.P. and a grant from the National Science Foundation (BNS-8912108) to E.Z.S

Correspondence should be addressed to Dr. John Walrond at the above address. Copyright (C) 1992 Society for Neuroscience $0270-6474 / 92 / 121490-12 \$ 05.00 / 0$
}

With few exceptions, the basic anatomy and physiology of invertebrate photoreceptors are similar. The structural organization of these cells plays a critical role in phototransduction by spatially constraining the sequence of reactions that modulate ion channels following light-induced isomerization of rhodop$\sin$. The transducing region of the cell bears millions of microvilli where the molecules for phototransduction (rhodopsin, G-protein, phospholipase C, etc.) initiate excitation and adaptation to light. The sequential activation of these molecules generates inositol- $(1,4,5)$-trisphosphate $\left(\operatorname{Ins}_{3}\right)$, which is thought to be the second messenger responsible for a light-induced elevation of $\mathrm{Ca}^{2+}$ (see reviews by Payne et al., 1988; Bacigalupo et al., 1990; Pak and Shortridge, 1991). Evidence for the involvement of the $\operatorname{Ins}_{3}$ pathway in invertebrate phototransduction comes primarily from electrophysiological studies on Limulus ventral photoreceptors (Brown et al., 1984; Brown and Rubin, 1984; Fein et al., 1984; Corson and Fein, 1987) and from biochemical studies on squid (Szuts et al., 1986; Brown et al., 1987; Baer and Saibil, 1988; Wood et al., 1989) and flies (Devary et al., 1987; Bloomquist et al., 1988).

In many transducing cells, Ins $\mathrm{P}_{3}$ releases calcium from an intracellular compartment, which in invertebrate photoreceptors is an extensive network of endoplasmic reticulum called submicrovillar cisternae (SMC) or palisade that lies immediately beneath the microvilli (Horridge and Barnard, 1965; Whittle, 1976; Calman and Chamberlain, 1982; reviewed by Walz and Baumann, 1989). In the retinular cells of leech, Limulus, and honeybee, SMC accumulates calcium by an ATP-dependent process (Walz, 1982; Walz and Fcin, 1983; Baumann and Walz, 1989b; Walz and Baumann, 1989) and releases calcium in response to $\mathrm{InsP}_{3}$ (Baumann and Walz, 1989b). SMC has been also implicated in the processing of newly synthesized and degraded microvillar membranes (reviews of Whittle, 1976; Blest, 1988). Thus, SMC appears to be an organelle with dual functions related to membrane renewal and calcium regulation.

The transducing microvilli of photoreceptors are segregated to specific regions of the cell. In cephalopods, this region is a dendrite-like process (200-500 $\mu \mathrm{m}$ long) called the distal segment that is nearly rectangular in cross-section. Two opposing surfaces of the distal segment are covered with densely packed microvilli, which are collectively called a rhabdomere for each surface. Because the biochemical and electrophysiological responses to light are similar in squid and Limulus (Pinto and Brown, 1977; Corson et al., 1983; Brown et al., 1984; Szuts et al., 1986; Baer and Saibil, 1987), cephalopod photorcceptors are expected to contain $\mathrm{Ca}^{2+}$-storage organelles similar to SMC. However, intracellular membrane systems that might be involved in calcium regulation or membrane turnover were rarely, 
if ever, observed in previous studies of distal segments (Zonana, 1961; Young, 1971; Cohen, 1973; Saibil, 1982; Robles et al. 1987; Saibil and Hewat, 1987).

The aim of our studies was to reexamine whether $\mathrm{Ca}^{2+}$-storage organelles exist in squid distal segments. Based on the hypothesis that the lack of observed intracellular organelles was due to a fixation problem stemming from poor penetration of fixative, we examined alternative fixation methods. Aldellyde fixation of $i$ solated receptor cells did not improve subcellular preservation even though the time for fixative penetration was extremely short. However, improved preservation and detection of membrane-bound compartments were obtained with the use of the ultra-rapid freezing technique. With this procedure, the vitread surface of isolated retinas was frozen against a liquid helium-cooled copper block. Preserving the distal ends of the photoreceptors proved advantageous for this study because photoisomerization preferentially occurs in distal microvilli where light is first absorbed in the living eye. The distal region also displays fewer membrane-bound organelles involved in rhabdom turnover, hecause such vesicles are predominantly located in the more proximal regions where they shuttle back and forth between the distal and proximal segments (Robles et al., 1984, 1987). For both of these reasons, the distal ends of the cells are expected to be enriched in cellular structures needed for phototransduction. Our results indicate that the distal segments of squid photoreceptors do not contain the prominent SMC seen in other invertebrates. Instead of cisternae, squid photoreceptors possess parallel rows of axially oriented tubes, which we call submicrovillar tubules (SMT).

Preliminary findings of these studies have been reported previously (Walrond and Szuts, 1989).

\section{Materials and Methods}

Animals. Live squid (Loligo pealei) were obtained from the Marine Resources Center of the Marine Biological Laboratory, and prior to each experiment, animals were dark adapted for $1 \mathrm{hr}$ in a light-tight bucket of sea water that was continuously bubbled with oxygen. The animals were decapitated and the retinas excised under dim red illumination.

Photoreceptor dissociation and fixation. Squid photoreceptors were dissociated by the protocol of Nasi and Gomez (in press). Isolated, darkadapted squid retinas were chopped into small pieces and incubated for $60 \mathrm{~min}$ at $10^{\circ} \mathrm{C}$ in oxygenated artificial sea water (ASW) containing $1 \%$ pronase (103 units $/ \mathrm{mg}$ protein; isolated from Streptomyces griseus, Calbiochem Corp., La Jolla, CA). Following incubation, retinal pieces were rinsed several times in ASW containing 3\% fetal calf serum, transferred to calcium-free ASW (no $\mathrm{CaCl}_{2}$ added) containing protease inhibitors ( $1 \mathrm{~mm}$ phenylmethylsulfonyl fluoride, $5 \mu \mathrm{g} / \mathrm{ml}$ leupeptin, $1 \mu \mathrm{g} / \mathrm{ml}$ pepstatin, and 0.25 inhibitory unit $/ \mathrm{ml} \alpha_{2}$-macroglobulin), and gently triturated for several minutes with a fire-polished Pasteur pipette. Undissociated tissue was removed and $10 \mathrm{~mm} \mathrm{CaCl}_{2}$ was added to the suspension. Dissociated photoreceptors were fixed for $30 \mathrm{~min}$ in $2 \%$ paraformaldehyde and $1 \%$ glutaraldehyde in $193 \mathrm{~mm} \mathrm{NaCl}, 12 \mathrm{~mm} \mathrm{KCl}, 20 \mathrm{~mm}$ $\mathrm{MgCl}_{2}, 26 \mathrm{~mm} \mathrm{MgSO} 4,10 \mathrm{~mm} \mathrm{CaCl}_{2}, 0.2 \mathrm{M} \mathrm{Na}$-cacodylate, pH 7.6; postfixed for $2 \mathrm{hr}$ with $1 \% \mathrm{OsO}_{4}$ in $0.1 \mathrm{~m}$ sodium phosphate buffer, $\mathrm{pH}$ 7.2; and centrifuged into a pellet that was block stained overnight with $1 \%$ uranyl acetate in $0.1 \mathrm{M} \mathrm{Na}$-acetate $(\mathrm{pH} 5.5$ ), dehydrated through a graded ethanol series, and embedded in Araldite.

Ulira-rapid freezing of intact retina. Excised retinas were placed in a shallow dish on ice and bathed in oxygen-saturated artificial seawater (480 mм NaCl, $10 \mathrm{~mm} \mathrm{KCl,} 49 \mathrm{~mm} \mathrm{MgCl}_{2}, 10 \mathrm{~mm} \mathrm{CaCl}_{2}, 10 \mathrm{~mm}$ HEPES, pH 7.8 with $\mathrm{NaOH}$ ). The retina was cut into $1 \mathrm{~mm}^{2}$ pieces, placed receptor side up on a $600-\mu \mathrm{m}$-thick slab of gelatin (15\% gelatin in sea water), and mounted on an aluminum planchet. The planchet was inserted into the freezing apparatus (Heuser et al., 1979), dropped onto a liquid helium-cooled copper block, and stored in liquid nitrogen. In most preparations the tips of the photoreceptors were well preserved for $20-30 \mu \mathrm{m}$ below the freezing surface.
Freeze-substitution, freeze-fracture, and freeze-etch. Ultra-rapidly frozen retinas were processed for freeze-substitution (Ornberg and Reese, 1980). Aluminum planchets were placed tissue side up on $5 \mathrm{ml}$ of a frozen solution of $4 \%$ osmium tetroxide in spectrophotometric grade acctonc, incubatcd at $-80^{\circ} \mathrm{C}$ ovcrnight, and gradually warmed to room temperature over $24 \mathrm{hr}$. Samples were block stained at room temperature with $20 \%$ uranyl acetate in methanol, followed by $0.25 \% \mathrm{HfCl}_{4}$ in acetone, and embedded in Araldite. Retinas were sectioned (0.25$0.5 \mu \mathrm{m}$ thick sections) for high-voltage electron microscopy $(1000 \mathrm{kV})$ or for standard electron microscopy $(0.05-0.075 \mu \mathrm{m}$ thin sections).

Alternatively, ultra-rapidly frozen retinas were freeze-fractured and etched for 10 min at $10^{-7}$ mbar at $-107^{\circ} \mathrm{C}$ in a Balzers 301 freezefracture device to sublimate the ice to a depth of 50-200 nm. The fractured and etched surfaces were rotary replicated with a 2-nm-thick layer of platinum-iridium-tantalum alloy followed by carbon (Bridgman et al., 1987).

\section{Results}

Chemical fixation. Squid retinas were dissociated and individual photoreceptors were chemically fixed to determine if improved access for aldehyde fixatives would improve the preservation of membrane-bound organelles. The dissociation protocol yielded a large number of photoreceptors that appeared intact from the axon hillock to the tip of the distal segment (Fig. 1). When viewed in thin sections cut parallel to the long axis of the cells, the distal segments displayed their characteristic architecture, showing a thin cytoplasmic layer sandwiched between two wide layers of rhabdomeres. However, membrane-bound organelles were found only rarely in these views regardless of the position along the length of the distal segment, and the cytoplasmic structure was usually disrupted (Fig. 2). Therefore, fixing isolated cells under our conditions did not improve ultrastructural preservation of these photoreceptors.

Rapid freezing. To minimize damage induced by chemical fixation, pieces of live squid retina were rapidly frozen against a liquid helium-coolcd coppcr block. The ultrastructure of rapidly frozen retinas is best preserved within $20-30 \mu \mathrm{m}$ of the extracellular matrix (the limiting membrane) that covers the retina at the distal tips of the photoreceptor cells. Thin sections of freeze-substituted retinas cut parallel to the long axis of the photoreceptors showed improved preservation of cytoplasmic structure compared to chemically fixed preparations. Although membrane-bound compartments were observed, most of these compartments appeared vesicular. Occasionally, the compartments appeared as saccules or flattened tubes located near the base of the microvilli and these saccules were separated from the plasma membrane along their entire length (Fig. 3).

The elongated shape of the saccules is more easily discerned in thicker sections (0.25-0.50 $\mu \mathrm{m}$ thick), shown in Figures 4 and 5 , that were also cut from rapidly frozen and freeze-substituted retinas. Because several microvilli are superimposed in a thick section, individual microvilli are more difficult to disccrn in these sections than in thin sections. Saccules, which are recognized as two membranes separated by a lumen, are seen in the most distal portions of the receptor cells adjacent to the limiting membrane (Fig. 4), but are more prominent along the rhabdomeric region (Fig. 5). The saccules run parallel to the rhabdomere and maintain a distance of about $40 \mathrm{~nm}$ (Table 1) from the plasma membrane at the base of the microvilli. In some cases, the saccule membranes appear crenulated and the lumen is not easily discerned (Fig. 5). Crenulation likely contributes to the predominately vesicular appearance of the saccules in thin sections since these membranes would tend to move in and out of the plane of a thin section. 

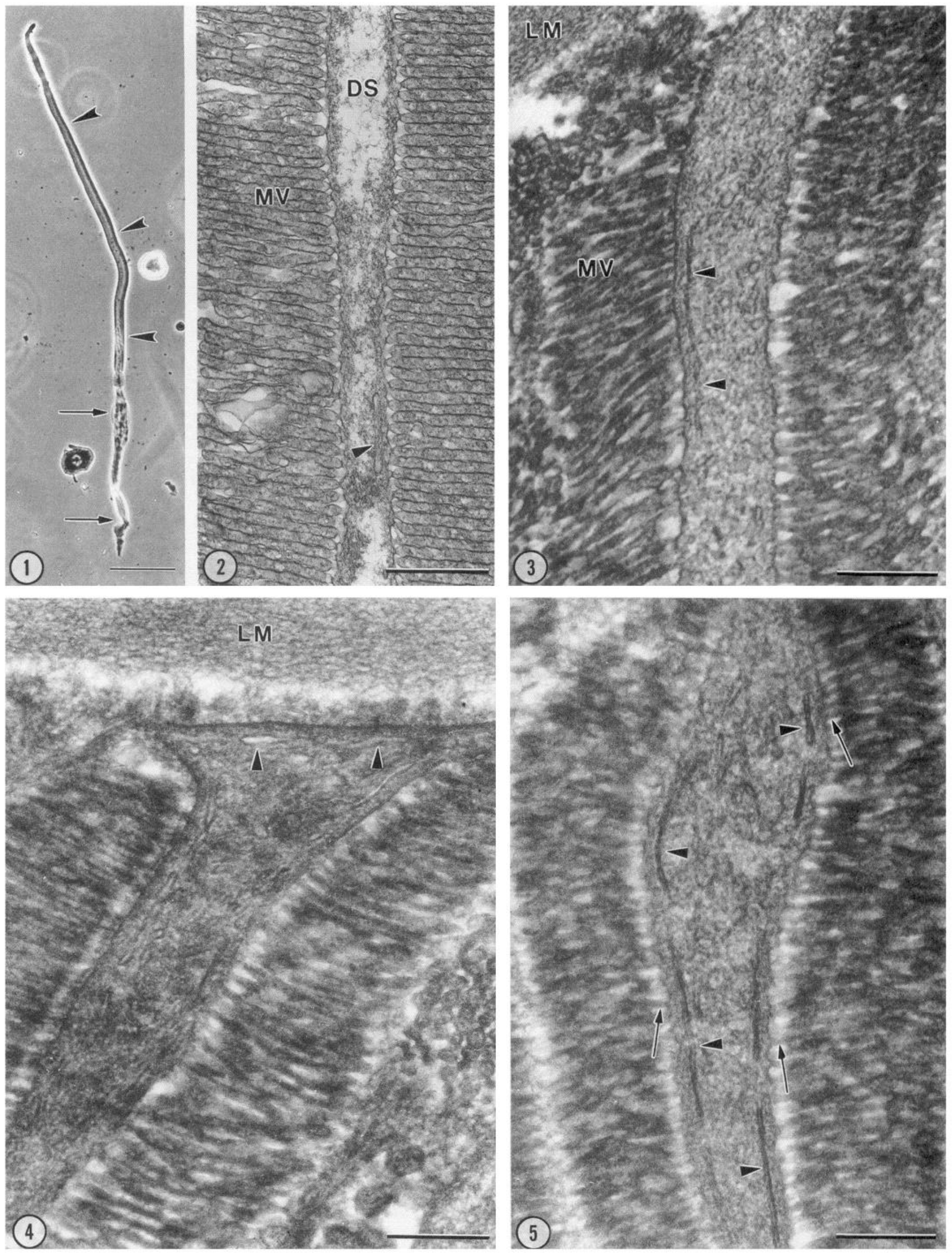
We also observed that in rapidly frozen preparations the bases of the microvilli appear distinctly different from those seen in chemically fixed cells. In freeze-substituted samples, microvilli gently taper to a narrow stalk at their base, the site of evagination from the plasma membrane. This microvillar stalk appears to be 50-70 $\mathrm{nm}$ long. In the sectioned, freeze-substituted preparations (Figs. 3-5), the stalk region gives rise to the apparent narrow, bright band that surrounds the cytoplasm and demarcates it from the heavily stained rhabdomeres. In contrast, the extent of the taper is greatly reduced for microvilli that were chemically fixed (Fig. 2) because such fixation conditions apparently induce swelling.

Freeze-fracture: organization of the rhabdomere. Photoreceptor cell topology was further investigated with freeze-fracture and etch techniques. These studies revealed several new features about the organization of squid rhabdomeres. Sublimation of ice from the extracellular space in freeze-fractured retinas revealed two types of connections (Figs. 6, 7). One type extends between the tips of the microvilli and the nonvillous surface of adjacent cells. These structures may be partly responsible for the organization of the retina since they likely contribute to the orthogonality of the rhabdomeres in neighboring cells. Dissociation of the retina into individual cells presumably requires breaking these connections. The second type of connection links adjacent villi and appears to constitute the "junctions" described for chemically fixed receptors (Saibil and Hewat, 1987). The microvillar diameter $(56 \mathrm{~nm}$; Table 1$)$ is less than the centerto-center spacing of the microvillar openings ( $63 \mathrm{~nm}$; Table 1), indicating that the observed gap between adjacent microvilli is 6-8 nm. Similar separations between microvilli were observed previously (Tsukita et al., 1988). The villar openings are quasihexagonally packed at a density of 245 per $\mu \mathrm{m}^{2}$ (Table 1). This measured value is in good agreement with the density calculated (252 per $\mu \mathrm{m}^{2}$ ) for a perfectly hexagonal lattice where the intermicrovillar distance is $63 \mathrm{~nm}$ (Table 1). Although the average packing is hexagonal when analyzed over a large area $\left(\approx 9 \mu \mathrm{m}^{2}\right)$, there are deviations from this pattern that can lead to localized square lattice packing (Fig. 8). A central filament, which appears to be the light-labile actin bundle described by others (Tsukita et al., 1988), is located in each cross-fractured and etched microvillus. The filaments are broken off where the microvilli open into the cytoplasm as seen in P-face views of the freeze-fractured basal plasma membrane of the rhabdomcres (Fig. 8). Thcir cxtension into the cytoplasm is limited to only a few nanometers heyond the basal membrane since their stubby ends are found within the narrow band of etched cytoplasm adjacent to the
Table 1. Dimensions of distal segment components

\begin{tabular}{lcr} 
Component & Mean $\pm \mathrm{SD}$ & $n$ \\
\hline $\begin{array}{l}\text { Submicrovillar tubules } \\
\quad \text { Diameter }\end{array}$ & $128 \pm 26 \mathrm{~nm}$ & $28(12)$ \\
$\quad \begin{array}{l}\text { Distance between plasma } \\
\quad \text { membrane and SMT }\end{array}$ & $42 \pm 15 \mathrm{~nm}$ & $70(6)$ \\
Microvilli & $56 \pm 10 \mathrm{~nm}$ & $100(4)$ \\
$\quad$ Diameter & $245 \mathrm{per} \mu \mathrm{m}^{2}$ & $(2)$ \\
$\quad$ Density & \\
$\quad$ Spacing & \\
Cytoskeletal elements (data corrected for $2 \mathrm{~nm}$ replica thickness) \\
$\quad$ Putative microtubule diameter & $24 \pm 2 \mathrm{~nm}$ & $16(3)$ \\
Actin-like filament diameter & $7 \pm 1 \mathrm{~nm}$ & $22(4)$
\end{tabular}

Data expressed as mean \pm SD for $n$ measurements; number of cells is in parentheses. Distance between plasma membrane and SMT was measured from thick sections of rapidly frozen retinas. All other data measured from freeze-fracture electron micrographs.

${ }^{a}$ Measured density was calculated from a count of 2105 microvillar openings over a total rhabdomeric area of $8.7 \mu \mathrm{m}^{2}$ from two cells.

${ }^{b}$ Measured as the distance between microvillar openings. A perfect hexagonal lattice with this mean \pm SD for spacing gives a density of $252 \pm 62$ microvilli per $\mu \mathrm{m}^{2}$.

fracture face (Figs. 8, 9). In this regard, the microvillar filaments of squid differ from the filaments seen in honeybee that extend morc than $0.5 \mu \mathrm{m}$ into the cytoplasm within fenestrations that penetrate the SMC (Baumann and Walz, 1989a).

Freeze-fracture: organization of the submicrovillar tubules. The irregular tube shape of the submicrovillar compartments, which appear as crenulated saccules in thick sections, becomes apparent in freeze-etched preparations (Fig. 8). In addition, the location of these compartments near the microvillar openings is confirmed in stereo views in which the fracture plane leaves the basal rhabdomeric membrane, passes through the cytoplasm at a shallow angle, and cross-fractures the tubules (Fig. 9). The tubular compartments are concentrated near the microvillar openings (Figs. 8, 9) and are in a position equivalent to the submicrovillar cisternae observed in arthropods and annelids. To conform with existing nomenclature, we call the structures in squid receptors SMT. Fracture faces and etched surfaces from individual tubules can be followed and counted to determine the number of membrancs comprising the organelle. The SMT is easily distinguishable from mitochondria since the plane of fracture needs to cross only two membranes to traverse the organelle. On entering the tubule, there is a single step onto the

$\leftarrow$

Figure 1. Light micrograph of an isolated, chemically fixed squid photoreceptor. The soma (lower arrow) appears bright in this Nomarski view. The proximal segment (upper arrow), also known as the inner segment, is the site of protein synthesis. The distal segment (arrowheads) is $280 \mu \mathrm{m}$ long and $7.5 \mu \mathrm{m}$ wide at its widest point including the microvilli, and is the site of phototransduction. Scale bar, $50 \mu \mathrm{m}$.

Figure 2. Thin section through the distal segment of a conventionally fixed photoreceptor from the same preparation shown in Figure 1. Microvilli $(M V)$ lie perpendicular to the axis of the distal segment $(D S)$. Cytoplasmic structure in the rhabdom is disrupted, and internal membrane-bound compartments are rarely observed (arrowhead). Scale bar, $0.5 \mu \mathrm{m}$.

Figure 3. Thin section of a rapidly frozen and freeze-substituted squid photoreceptor from an intact retina. A membrane-bound compartment (arrowheads) located at the base of the microvilli (MV) extends along the long axis of the cell parallel to the rhabdom. The limiting membrane $(L M)$ lies within $2-4 \mu \mathrm{m}$ of the freezing surface. Scale bar, $0.5 \mu \mathrm{m}$.

Figure 4. Thick section $(0.25-0.33 \mu \mathrm{m})$ of a rapidly frozen and freeze-substituted squid photoreceptor from an intact retina. Flattened membranebound compartments (arrowheads) extend to the distal tips of the photoreceptors and can be also found beneath the nonvillous plasma membrane that contacts the limiting membrane $(L M)$. Scale bar, $0.5 \mu \mathrm{m}$.

Figure 5. Thick section of a rapidly frozen and freeze-substituted photoreceptor located farther from the limiting membrane than the one shown in Figure 4. The membrane-bound compartments (arrowheads) are flattened tubules that extend along the length of the distal segment and run parallel to the rhabdom near the base of the microvilli (arrows). Scale bar, $0.5 \mu \mathrm{m}$. 

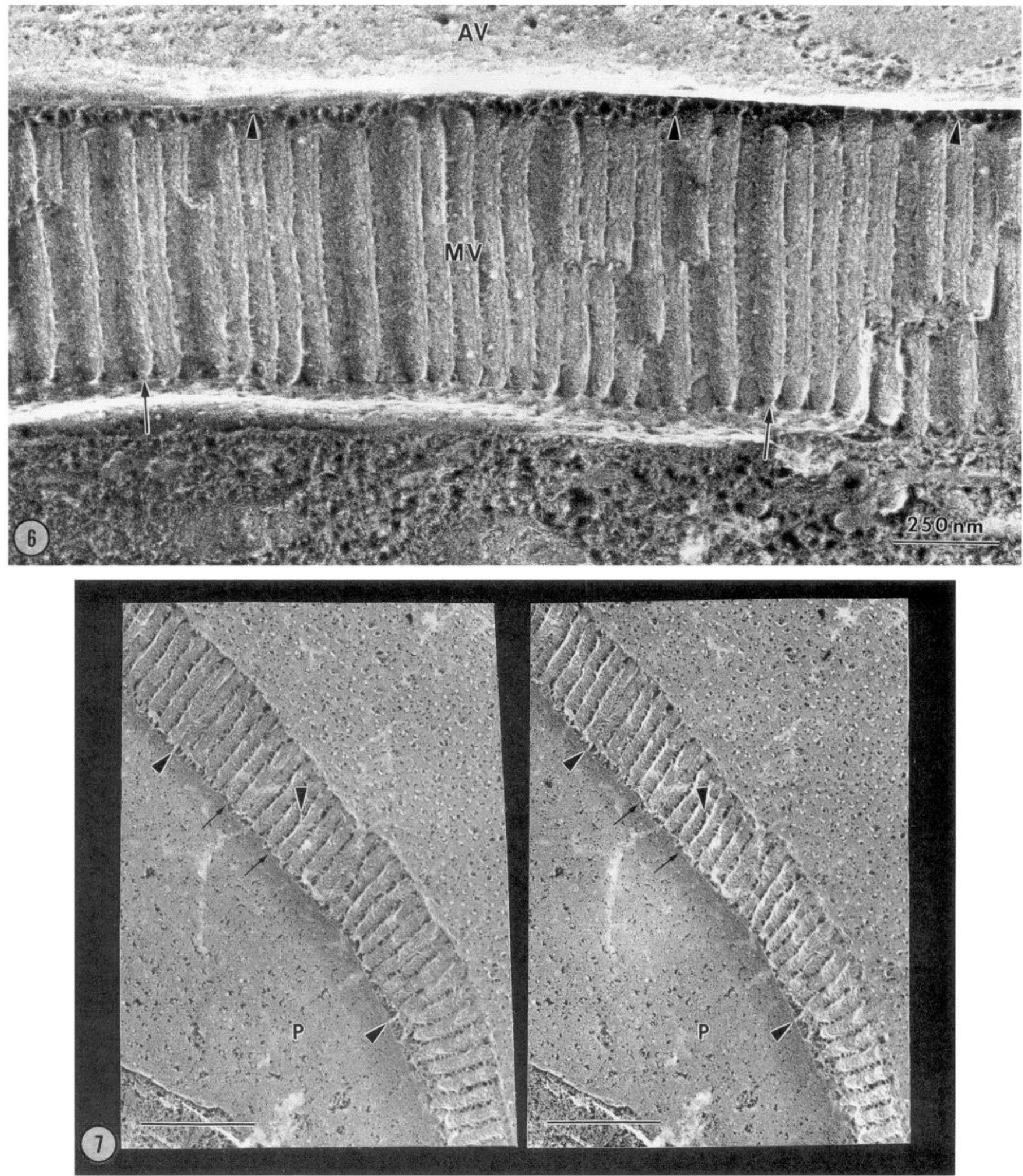

Figure 6. Freeze-fractured and etched microvilli. Extracellular connections (arrowheads) span the extracellular space between the microvilli ( $M V$ ) and the avillar portion $(A V)$ of a neighboring photoreceptor. Microvilli are arranged in a regular pattern and lie parallel to one another. The rhabdomeric E-face and the microvilli are continuous at the microvillar bases (arrows). Scale bar, $250 \mathrm{~nm}$.

Figure 7. Stereo view of freeze-fractured and etched microvilli. Each microvillus is separated from its neighbor by a narrow space, which here is larger than the average. Extracellular connections (arrowheads) span the gaps between microvilli and between microvilli and the avillar surface of an adjacent photoreceptor. The P-face $(P)$ and the extracellular surface of the adjacent cell are separated by a shallow step (arrows). Scale bars, $0.5 \mu \mathrm{m}$. 

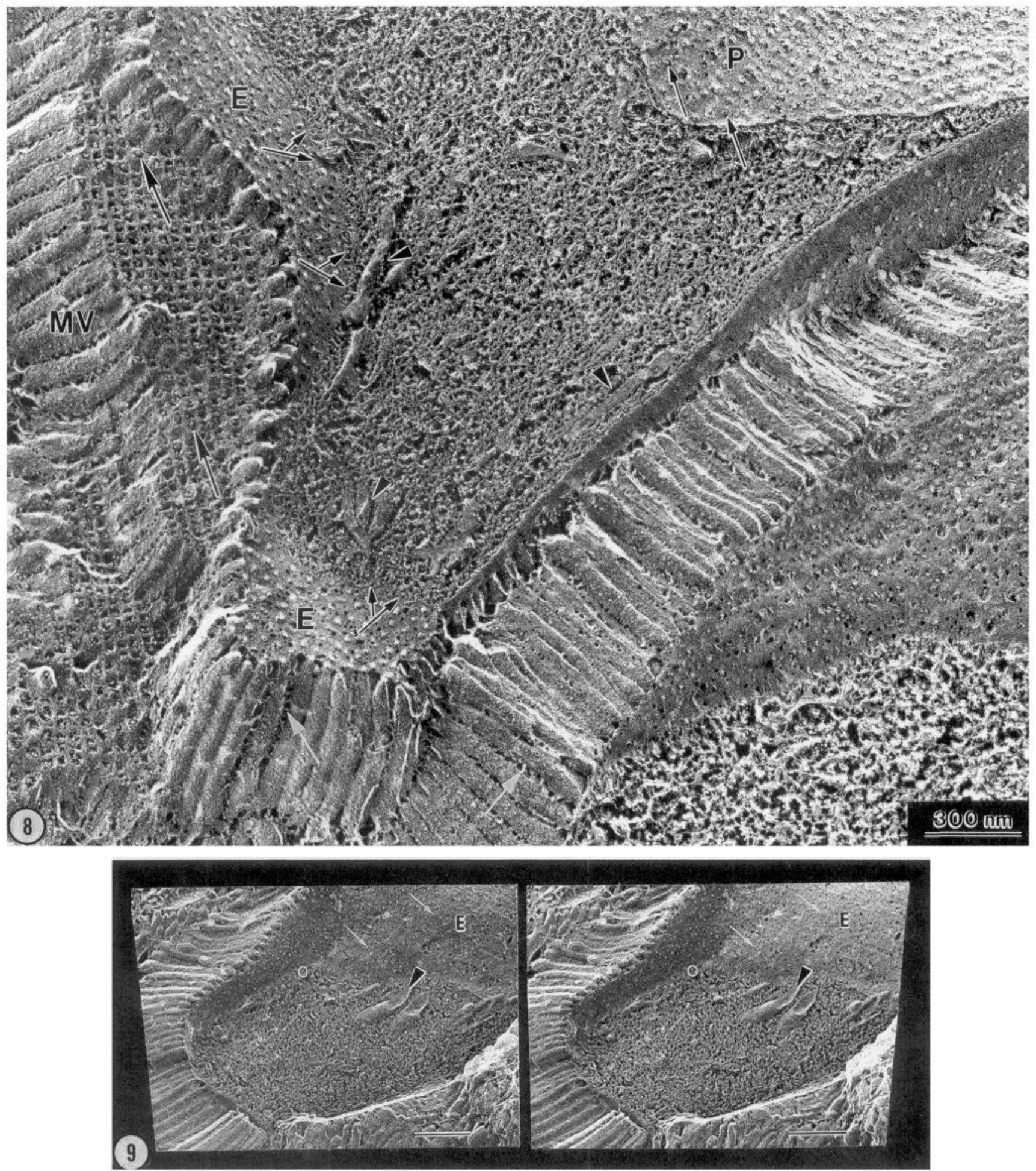

Figure 8. Freeze-fracture and etch view of membrane and cytoplasm in a rapidly frozen photoreceptor. A central filament (large solid arrows) is located in each of the cross-fractured microvilli $(M V)$ and extends into the plasmalemmal E-face $(E)$; broken-off microvillar filaments produce a pattern of bright knobs in the E-face. In the P-face $(P)$, the filament (small solid arrows) nearly fills the microvillar opening. A dense meshwork of cytoskeletal elements separates the SMT (arrowheads) from the inner surface of the plasmalemma (double-headed solid arrows). Extracellular connections (open arrows) extend between adjacent microvilli. Scale bar, $300 \mathrm{~nm}$.

Figure 9. Stereo pair of a freeze-fractured and etched photoreceptor. Stubs of the microvillar filaments (arrows) are visible in the plasmalemmal E-face $(E)$. The filaments are found in the cytoskeletal meshwork (circle) only in the thin layer of cytoplasm near the membrane inner surface. The plane of fracture passes obliquely through the cytoplasm to reveal the P-and E-fracture faces and the true cytoplasmic surface of an SMT (arrowhead) that is located near the microvillar openings. Scale bars, $0.5 \mu \mathrm{m}$. 

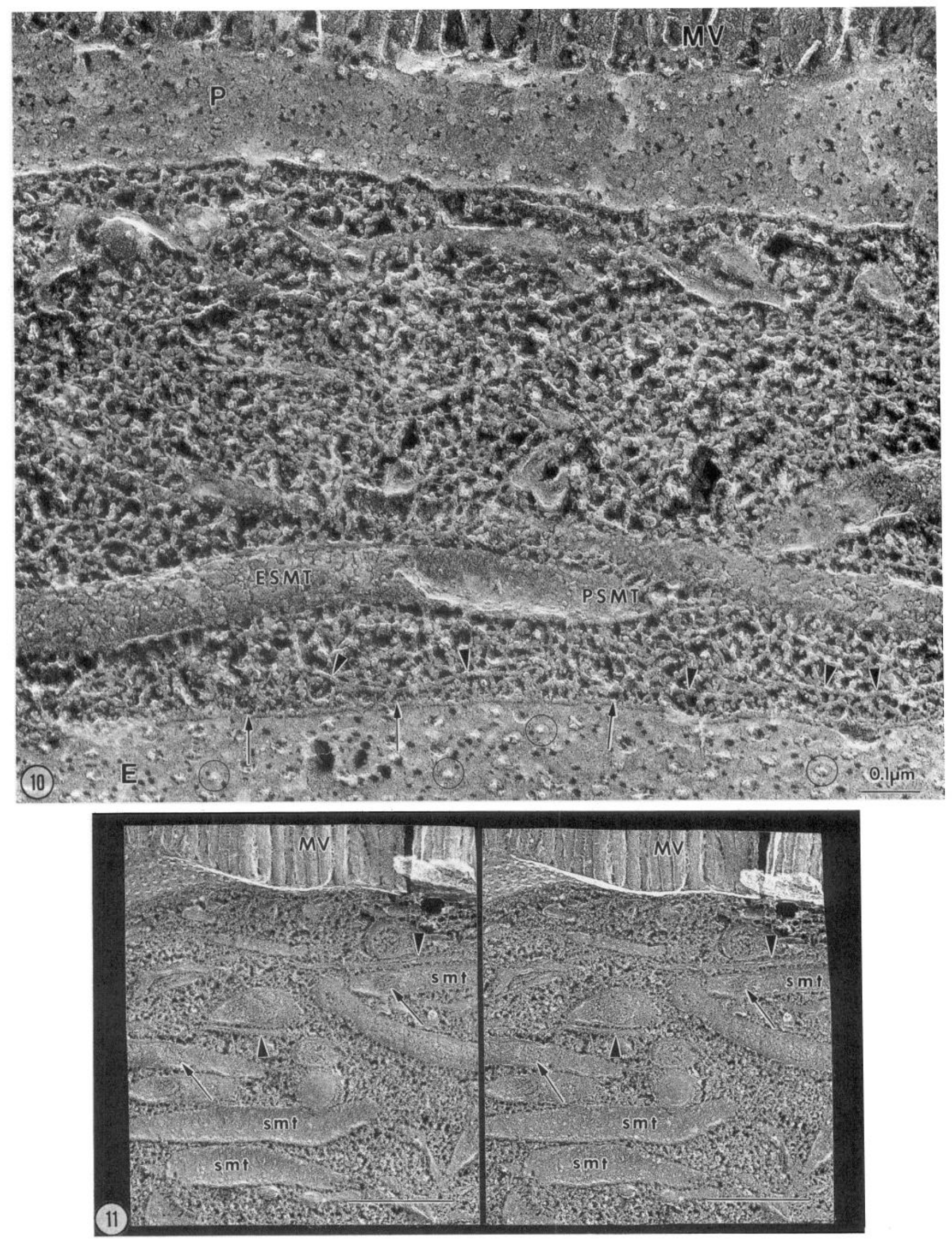

Figure 10. Freeze-fractured and etched view of photoreceptor cytoskeleton and an SMT showing its tubular shape. Cross-fractured tubules contain only one E-face (ESMT) and one P-face (PSMT). A meshwork of cytoskeletal filaments connects the SMT and the inner surface of the plasmalemma 


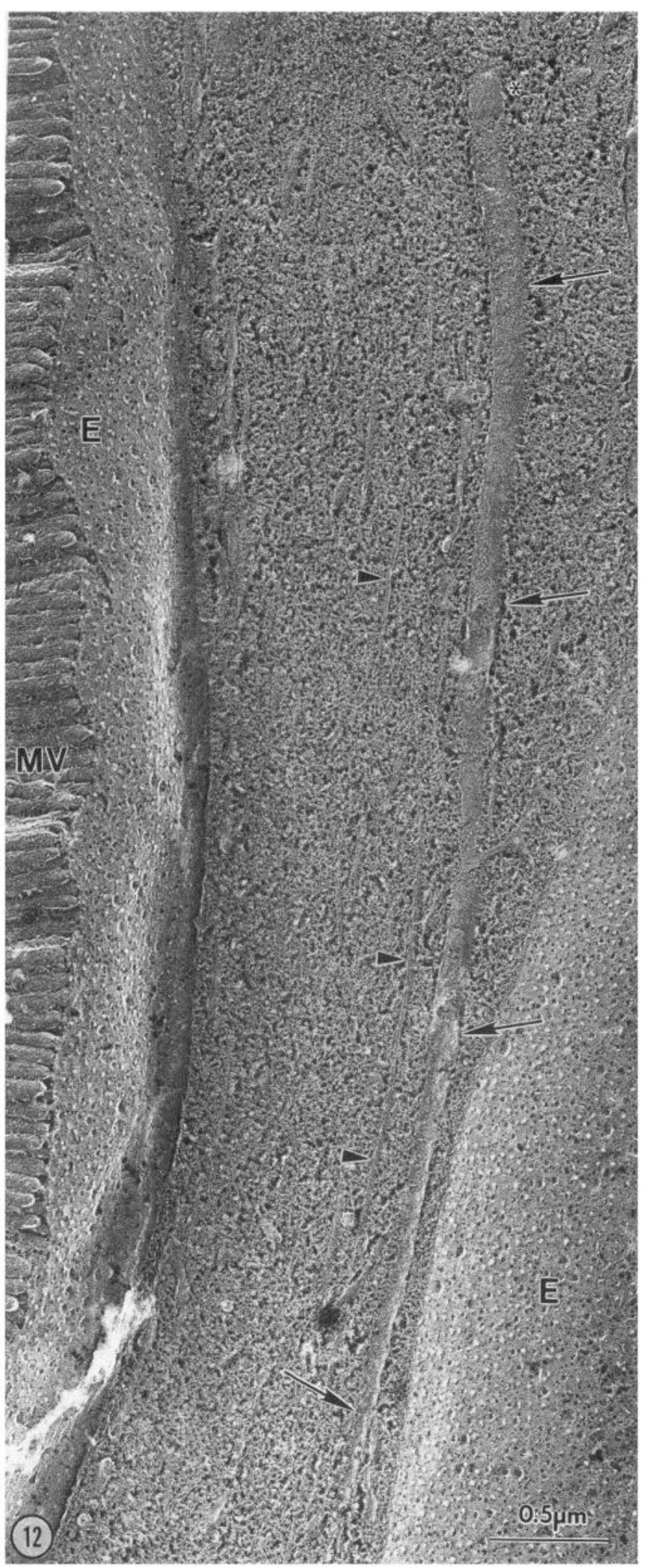

SMT P-face as the fracture plane leaves the cytoplasm. A subsequent small step between the P-face and the cross-fractured lumenal contents exposes the lumenal inner surface. The fracture plane then skips to the E-face of the tubule and finally to its outer surface before it meanders into the filamentous network of the cytoplasm (Figs. 9-11). Measurements indicate that the tubules are about $130 \mathrm{~nm}$ in diameter (Table 1). We could not determine the axial distance extended by the tubules, because the rapid freezing techniques used in this study preserved only the distal $20-30 \mu \mathrm{m}$ of the photoreceptors. However, individual tubules could be followed for as long as $6 \mu \mathrm{m}$ (Fig. 12). Although $6 \mu \mathrm{m}$ is a relatively short distance, corresponding to about $2 \%$ of the length of the distal segment, the tubules are likely to be much longer and may extend over a substantial length of the rhabdomere.

The SMT are embedded in a network of cytoskeletal elements that includes putative actin filaments and microtubules (Figs. 10-12). The filaments and microtubules are predominantly oriented parallel to the SMT and to the basal rhabdomeric membrane. The observed diameters for the microtubules and actin-like filaments in Figure 10 are about $24 \mathrm{~nm}$ and $7 \mathrm{~nm}$, respectively (Table 1), after the measured values are corrected for the $2 \mathrm{~nm}$ thickness of the platinum replica. The observed microtubules are likely to be involved in the light-dependent movement of screening pigments (Daw and Pearlman, 1974). They may also mediate movement of the SMT if the prediction by Whittle (1976) that endoplasmic reticulum is capable of photomechanical responses within squid distal segments is correct. In this study, we did not investigate the role of light adaptation or circadian rhythms on the cellular distribution of SMT.

\section{Discussion}

Organization of submicrovillar tubules and rhabdom. Our results indicate that the putative $\mathrm{Ca}^{2+}$-storage organelles in the distal segments of cephalopod photoreceptors are elongated, 130-nmdiameter tubules, which we call SMT. Discerning the nature of these tubes required the use of ultra-rapid freezing since chemically fixed receptors contained few organelles that could be classified as membrane-bound compartments. These findings are consistent with previous studies on chemically fixed cephalopod photoreceptors that showed a paucity of well-preserved organelles (Zonana, 1961; Young, 1971; Cohen, 1973; Saibil, 1982; Saibil and Hewat, 1987). In contrast, ultra-rapidly frozen and freeze-substituted photoreceptors contained numerous ve-

Figure 12. The SMTs extend for long distances parallel to the rhab-
dom. A fractured and etched tubule (arrows) is visible over a distance
of about $6 \mu \mathrm{m}$. The E-faces $(E)$ of the rhabdom are visible on both sides
of the cross-fractured and etched cytoplasm. The distal end of the tubule
is cross-fractured $(*)$, and the proximal end was torn away by the frac-
ture, indicating that individual tubules may extend for distances greater
than $6 \mu \mathrm{m}$. Microtubules (arrowheads) are predominantly located in the
more central region of the cell. $M V$, microvilli. Scale bar, $0.5 \mu \mathrm{m}$.

(arrows). The width of some of the filaments (arrowheads) running parallel to the SMT and plasma membrane suggests that they are actin. The plasmalemmal E-face $(E)$ contains stubs of the microvillar filaments (circles). Microvilli $(M V)$ continuous with the plasmalemmal P-face $(P)$ are located at the top. Scale bars, $100 \mathrm{~nm}$.

Figure 11. Stereo view of freeze-fractured and etched submicrovillar tubules. The tubules (smt) and microtubules (arrowheads) run parallel to the rhabdom. Cross-fractures through the tubules show a nonetchable granularity in the tubule lumen (arrows). Scale bar, $0.5 \mu \mathrm{m}$. 


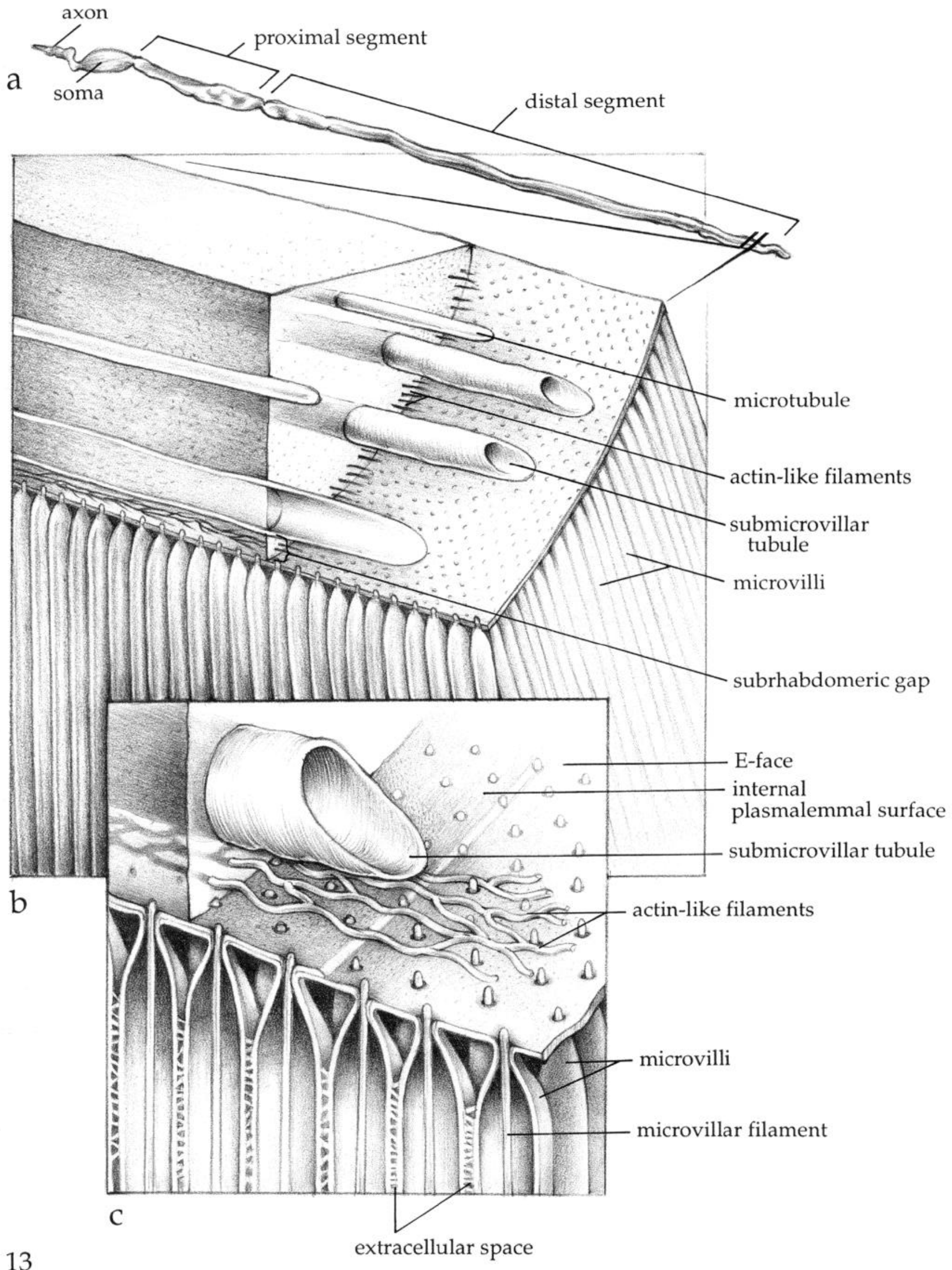


sicular profiles when viewed in thin sections $(0.050-0.075 \mu \mathrm{m}$ thick). For a submicrovillar tubule to extend for an appreciable distance in a thin section, the plane of section must be nearly parallel to the axis of the tubule. Therefore, the predominantly vesicular appearance in such sections is attributable to both the thinness of the section and the angle of the cut relative to the axis of the tubule. The elongated, saccule-shape of the SMT was more detectable in thick sections $(0.25-0.50 \mu \mathrm{m})$, where the crenulated tubes tended to remain in the plane of the section over longer distances.

Without ctching, freczc-fracturcd photoreceptors provided little information about the shape and distribution of the SMT since the fracture plane usually followed the tubule membrane for only a short distance before entering the cytoplasm. Sublimation of ice from fractured surfaces exposed the SMT and showed that the putative $\mathrm{Ca}^{2+}$-storage organelles are individual tubes, corresponding to the flattened saccules observed in thick sections. The freeze-fracture and etch views also showed that the tubules are located near the base of the microvilli and are situated appropriately to interact with any second messenger (such as $\mathrm{InsP}_{3}$ ) that diffuses out of the microvillar openings. The contents of cross-fractured tubules did not entirely sublimate (see Fig. 9), suggesting that the tubules contain proteinaceous material.

The organization of the SMT in the distal segment is summarized schematically in Figure 13. Squid photoreceptors are elongated cells that contain two microvillus-bearing surfaces on their distal segments (Fig. 13a). The SMT and microtubules are preferentially oriented parallel to the long axis of the distal segment. A 40-nm-wide gap separates the SMT from the basal rhabdomeric plasma membrane, and this gap contains putative actin filaments that run parallel to the SMT and plasma membrane (Fig. 13b,c). In chemically fixed preparations that have been described previously (Saibil and Hewat, 1987), plasma membranes of adjacent microvilli abut one another so that away from the loop region extracellular space is occluded along the length of microvilli. In contrast, the freeze-fracture and etch views indicate that the microvilli are separated by a narrow extracellular space that is $6-8 \mathrm{~nm}$ wide. This width is sufficiently large to permit exchange of extracellular ions bathing the entire villar surface. The space between microvilli is clearly not a sign of rhabdomeric breakdown. Disordered microvilli were not observed in our samples, just as they were absent in the study of Tsukita et al. (1988). Measurements of the distances between microvillar openings in the basal rhabdomeric membrane indicate that the observed microvilli retain their quasihexagonal packing since the openings are regularly spaced about $63 \mathrm{~nm}$ apart. Although our measurements were made only on the distal ends of the receptors, we propose that observed separations between microvilli are representative for the entire rhabdom. The apposition of adjacent microvillar membranes reported by Saibil and Hewat (1987) was based on chemically fixed retinas, and chemical fixation appears to cause artifactual swelling of cephalopod photoreceptors. Because ultra-rapid freezing leads to better cellular preservation, the observed spaces between the microvilli are more representative of the living state of squid photoreceptors.

The orientation and diameter of the tubules limit the number that can fit around the cell circumference and still remain within $\approx 40 \mathrm{~nm}$ from the rhabdomere. At maximum, six tubules (128 $\mathrm{nm}$ diameter) can fit within each $1 \mu \mathrm{m}$ of receptor cell circumference since the space between tubules exceeds $35 \mathrm{~nm}$. Since the portion of the distal scgment circumference containing microvilli is typically about $10 \mu \mathrm{m}$ (Cohen, 1973; Tsukita et al., 1988), a photoreceptor could contain a maximum of 60 tubules adjacent to the basal plasma membrane. This is an estimate since we did not obtain views that would have permitted a direct count. The volume of the subrhabdomeric gap (formed between the SMT and basal plasma membrane) is so small that small changes in the number of free ions can produce large changes in ionic concentration. For example, the release of 3000 calcium ions (or InsP $\mathrm{P}_{3}$ molecules) into the gap beneath a $1 \mu \mathrm{m}^{2}$ rhabdomeric area would yield a concentration of $100 \mu \mathrm{M}$ in the absence of adsorption to binding sites. Therefore, the location of the SMT near the microvillar openings and the relatively constant width of the subrhabdomeric gap $(\approx 40 \mathrm{~nm})$ is appropriate for these tubules to function in the uptake and release of calcium in the light response.

Comparisons with submicrovillar cisternae. In cellular organization, the submicrovillar tubules of squid clearly differ from the submicrovillar cisternae of annelids and arthropods. The most striking feature of cisternae is that they consist of large convoluted compartments that are positioned in an $\approx 1-\mu \mathrm{m}$ deep band beneath the rhabdomere. The cellular organization of the SMC has been studied most extensively in the honeybee drone using standard chemical fixation techniques (Skalska-Rakowska and Baumgartner, 1985; Baumann and Walz, 1989a). Based on the use of conventional thin sections, these studies showed that the SMC extend throughout the entire photoreceptor, occupy a large volume of the cell, and are topologically continuous with the endoplasmic reticulum. The SMC often appear as a palisade near the basal plasma membrane and frequently contain vacuole-like enlargements. A 200-500-nm-wide cytoplasmic gap separates most of the SMC from the rhabdom, but localized cxtensions of the SMC reduce this gap to $20-40$ $\mathrm{nm}$. The cytoplasmic gap at a nonvillous plasma membrane may be as narrow as $20-25 \mathrm{~nm}$.

The limited region of good preservation allowed by ultrarapid freezing prevented us from studying the topology of the SMT throughout the distal segment and determining its relationship to the endoplasmic reticulum in the rest of the cell. However, our observation that individual tubules are continuous over at least a $6 \mu \mathrm{m}$ length of the distal segment is consistent with the possibility that SMT, just as SMC, forms a continuous

Figure 13. Schematic representation of a squid photoreceptor cell, drawn nearly to scale. $a$, View of a photoreceptor cell with its soma, short proximal segment, and long distal segment. The phototransducing microvilli comprising the two rhabdomeres on each cell are located exclusively on the distal segment. $b$ and $c$, Increasingly enlarged views of a subrhabdomeric region. The SMTs extend to the tip of the distal segment and are located near the basal plasma membrane of each rhabdomere. The subrhabdomeric gap between the tubules and the basal plasmalemma contains actin-like filaments that run parallel to the axis of both the receptor cell and the SMT. Microtubules, which are also oriented parallel to the axis of the distal segment, are predominantly located in the more central cytoplasm. $c$, The microvillar filament extends through the E-leaflet of the plasma membrane and projects into the cortical cytoplasm for only a short distance so that it does not reach the SMT. The microvilli taper near their bases where they fuse with the plasma membrane. A narrow channel of extracellular space, which is spanned by linkages, separates adjacent microvilli and provides access of the extracellular medium to the entire receptor membrane surface. 
membrane system. The existence of such a continuum was predicted for squid (Whittle, 1976).

In several arthropods, SMC is considered to be involved in both calcium regulation and microvillar membrane turnover. SMT may also perform both of these functions. Clearly, our morphological studies cannot resolve which of these functions is primarily attributable to SMT. Nevertheless, the position of the SMT near the base of the microvilli and the relatively constant width of the subrhabdomeric gap suggest that SMT in squid serve the same function(s) as the submicrovillar cisternae of arthropods and annelids.

Why do squid receptors lack the prominent SMC organization? One possible explanation is that cephalopods do not use intracellular stores of calcium for phototransduction but depend on calcium influx from the extracellular space. Although InsP $_{3}$ can modulate calcium channels in plasma membranes (e.g., Kuno and Gardner, 1987), this form of calcium channel regulation has not yet been demonstrated for any photoreceptor. A more likely explanation is that the cytoplasm of distal segments cannot accommodate the relatively large cisternae characteristic of arthropod retinular cells. Most of the volume of squid distal segments is occupied by $\approx 1-\mu \mathrm{m}$-long microvilli, and only a narrow (0.5- $\mu \mathrm{m}$-wide) tongue of cytoplasm separates the rhabdomeres (Figs. 2-5). Thus, no space is available for extensively pleated cisternae beneath each rhabdomere. The physiological need for a voluminous intracellular compartment is also less essential for cephalopods since the volume-to-surface ratio of cytoplasm that is surrounded by microvilli is at least an order of magnitude smaller in squid distal segments than in the corresponding segments (R-lobes) of Limulus ventral photoreceptors (Limulus data from Calman and Chamberlain, 1982). Therefore, the calcium-regulating organelle could be commensurately smaller in cephalopods and still be capable of operating within the range of concentrations that exist for calcium and $\operatorname{InsP}_{3}$ in arthropod receptor cells.

In summary, we used ultra-rapid freezing, freeze-fracture, and freeze-substitution techniques to demonstrate the existence of membrane-bound organelles likely to be involved in cephalopod phototransduction. We found that the organization of cephalopod photoreceptors differs from other invertebrate photoreceptor cells in that cephalopods lack voluminous, highly convoluted SMC. Instead, cephalopod photoreceptors contain elongated membrane-bound tubules that are preferentially positioned beneath the rhabdom. The location of these tubules at a constant distance from the microvillar openings $(\approx 40 \mathrm{~nm})$ suggests that they are functionally equivalent to the cisternae of arthropods and annelids. Specifically, they are prime candidates for being the compartments that store and release calcium in response to the light-mediated formation of inositol trisphosphate.

\section{References}

Bacigalupo J, Johnson E, Robinson P, Lisman JE (1990) Second messengers in invertebrate phototransduction. In: Transduction in biological systems (Hidalgo $\mathbf{C}$, Bacigalupo $\mathrm{J}$, Jaimovich $\mathbf{E}$, Vergara $\mathbf{J}$, eds), pp 27-45. New York: Plenum.

Baer KM, Saibil HR (1988) Light- and GTP-activated hydrolysis of phosphatidyl-inositol bisphosphate in squid photoreceptor membranes. J Biol Chem 263:17-20.

Baumann O, Walz B (1989a) Topography of $\mathrm{Ca}^{2+}$-sequestering endoplasmic reticulum in photoreceptors and pigmented glial cells in the compound eye of the honeybee drone. Cell Tissue Res 255:511522.
Baumann O, Walz B (1989b) Calcium- and inositol polyphosphatesensitivity of the calcium-sequestering endoplasmic reticulum in the photoreceptor cells of the honeybee drone. J Comp Physiol A 165: $627-636$

Blest AD (1988) The turnover of phototransductive membrane in compound eyes and ocelli. Adv Insect Physiol 20:1-53.

Bloomquist BT, Shortridge RD, Schneuwly S, Perdew M, Montell C, Steller I1, Rubin G, Pak WL (1988) Isolation of a putative phospholipase C gene of Drosophila, norp A, and its role in phototransduction. Cell 54:723-733.

Bridgman PC, Carr C, Pedersen SE, Cohen JB (1987) Visualization of the cytoplasmic surface of Torpedo postsynaptic membranes by freeze-etch and immunoelectron microscopy. J Cell Biol 105:18291846.

Brown JE, Rubin LJ (1984) A direct demonstration that inositol trisphosphate induces an increase in intracellular calcium in Limulus photoreceptors. Biochem Biophys Res Commun 125:1137-1142.

Brown JE, Rubin LJ, Ghalayini AJ, Tarver AP, Irvine RF, Berridge MJ, Anderson RE (1984) Myoinositol polyphosphate may be a messenger for visual excitation in Limulus photoreceptors. Nature 311:160-163.

Brown JE, Watkins DC, Malbon CC (1987) Light-induced changes in the content of inositol phosphates in squid (Loligo pealei) retina. Biochem J 247:293-297.

Calman BG, Chamberlain SC (1982) Distinct lobes of Limulus ventral photoreceptors. II. Structure and ultrastructure. J Gen Physiol 80: $839-862$

Cohen AI (1973) An ultrastructural analysis of the photoreceptors of the squid and their synaptic connectiol.s. J Comp Neurol 147:351378.

Corson DW, Fein A, Walthall W (1983) Chemical excitation of $L i$ mulus photoreceptors. II. Vandate, GTP- $\gamma-\mathrm{S}$ and fluoride prolong excitation evoked by dim flashes of light. J Gen Physiol 82:659-667.

Corson DW, Fein A (1987) Inositol 1,4,5-trisphosphate induces bursts of calcium release inside Limulus ventral photoreceptors. Brain Res 423:343-346.

Daw NW, Pearlman AL (1974) Pigment migration and adaptation in the eye of the squid, Loligo pealei. J Gen Physiol 63:22-36.

Devary O, Heichal O, Blumenfeld A, Cassel D, Suss E, Barash S, Rubinstein CT, Minke B, Selinger Z (1987) Coupling of photoexcited rhodopsin to inositol phospholipid hydrolysis in fly photoreceptors. Proc Natl Acad Sci USA 84:6939-6943.

Fein A, Payne R, Corson DW, Berridge MJ, Irvine RF (1984) Photoreceptor excitation and adaptation by inositol 1,4,5-trisphosphate. Nature 311:157-160.

Heuser JE, Reese TS, Dennis MJ, Jan Y, Jan L, Evans L (1979) Synaptic vesicle exocytosis captured by quick freezing and correlated with quantal transmitter release. J Cell Biol,81:275-300.

Horridge GA, Barnard PBT (1965) Movement of palisade in locust retinula cells when illuminated. Q J Microsc Sci 106:131-135.

Kuno M, Gardner P (1987) Ion channels activated by inositol-1,4,5trisphosphate in plasma membrane of humnan T-lymphocytes. $\mathrm{Na}$ ture 326:301-304.

Nasi E, Gomez M (1992) Electrophysiological recordings in solitary photoreceptors from the retina of the squid, Loligo pealei. Visual Neurosci, in press.

Ornberg RL, Reese TS (1980) A freeze-substitution method for localizing divalent cations: examples from secretory systems. Fed Proc 39:2802-2808.

Pak WL, Shortridge RD (1991) Inositol phospholipid and invertebrate photoreceptors. Photochem Photobiol 53:871-875.

Payne R, Walz B, Levy S, Fein A (1988) The localization of calcium release by inositol trisphosphate in Limulus photoreceptors and its control by negative feedback. Philos Trans R Soc Lond [Biol] 320: 359-379.

Pinto LH, Brown JE (1977) Intracellular recordings from photoreceptors of the squid (Loligo pealei). J Comp Physiol 122:241-250.

Robles LJ, Cabebe CS, Aguilo JA, Anyakora PA, Bok D (1984) Autoradiographic and biochemical analysis of photoreceptor renewal in Octopus retina. J Neurocytol 13:145-164.

Robles LJ, Watanabe A, Kremer NE, Wong F, Bok D (1987) Immunocytochemical localization of photopigments in cephalopod retinae. J Neurocytol 16:403-415.

Saibil H, Hewat E (1987) Ordered transmembrane and extracellular structure in squid photoreceptor microvilli. J Cell Biol 105:19-28. 
Saibil HR (1982) An ordered membrane-cytoskeleton network in squid photoreceptor microvilli. J Mol Biol 158:435-456.

Skalsa-Rakowska JM, Baumgartner B (1985) Longitudinal continuity of the subrhabdomeric cisternae in the photoreceptors of the compound eye of the drone. Fxperientia 41:43-45.

Szuts EZ, Wood SF, Reid MS, Fein A (1986) Light stimulates the rapid formation of inositol trisphosphate in squid retinas. Biochem J 240:929-932.

Tsukita S, Tsukita S, Matsumoto G (1988) Light-induced structural changes of cytoskeleton in squid photoreceptor microvilli detected by rapid-freeze method. J Cell Biol 106:1151-1160.

Walrond JP, Szuts EZ (1989) Putative calcium stores within distal segments of squid photoreceptors. Biophys J 55:60a.

Walz B (1982) $\mathrm{Ca}^{2+}$-sequestering smooth endoplasmic reticulum in an invertebrate photoreceptor. I. Intracellular topography as revealed by OsFeCN staining and in situ $\mathrm{Ca}$ accumulation. J Cell Biol 93:839848.
Walz B, Baumann O (1989) Calcium sequestering cell organelles: in situ localization, morphological and functional characterization. Prog Histochem Cytochem 20:1-45.

Walz B, Fein A (1983) Evidence for calcium-sequestering smooth ER in I.imulus ventral photoreceptors. Invest Ophthalmol Vis Sci 24: 281.

Whittle AC (1976) Reticular specializations in photoreceptors: a review. Zool Scr 5:191-206.

Wood SF, Szuts EZ, Fein A (1989) Inositol trisphosphate production in squid photoreceptors: activation by light, aluminum fluoride and guanine nucleotides. J Biol Chem 264:12970-12976.

Young JZ (1971) The anatomy of the nervous system of Octopus vulgaris. Oxford: Oxford UP.

Lonana HV (1961) Fine structure of squid retina. Bull Johns Hopkins Hosp 109:185-205. 